\title{
Pulmonary IL-33 orchestrates innate immune cells to mediate respiratory syncytial virus-evoked airway hyperreactivity and eosinophilia
}

\author{
Yi-Hsiu Wu ${ }^{1,2}$ (1) | Alan Chuan-Ying Lai ${ }^{2}$ | Po-Yu Chi ${ }^{2}$ | Christina Li-Ping Thio ${ }^{2}$ | \\ Wei-Yu Chen ${ }^{3}$ | Ching-Hui Tsai ${ }^{4}$ (D) | Yungling Leo Lee ${ }^{2,4}$ (D) | Nicholas W. Lukacs ${ }^{5}$ | \\ Ya-Jen Chang ${ }^{1,2}$ (D)
}

\footnotetext{
${ }^{1}$ Taiwan International Graduate Program in Molecular Medicine, National Yang-Ming University and Academia Sinica, Taipei, Taiwan

${ }^{2}$ Institute of Biomedical Sciences, Academia Sinica, Taipei, Taiwan

${ }^{3}$ Institute for Translational Research in Biomedicine, Chang Gung Memorial Hospital, Kaohsiung, Taiwan

${ }^{4}$ Institute of Epidemiology and Preventive Medicine, National Taiwan University, Taipei, Taiwan

${ }^{5}$ Department of Pathology, University of Michigan, Ann Arbor, MI, USA

\section{Correspondence}

Ya-Jen Chang, Institute of Biomedical Sciences, Academia Sinica, No. 128 Academia Road, Section 2, Nankang, Taipei 11529, Taiwan

Email: yajchang@ibms.sinica.edu.tw

\section{Funding information}

This work was supported in part by the Ministry of Science and Technology [105-2628-B-001-009-MY3 to YJC] and by the Academia Sinica Career Development Award [104-CDA-L05 to YJC] in Taiwan.
}

\begin{abstract}
Background: Respiratory syncytial virus (RSV) infection is epidemiologically linked to asthma. During RSV infection, IL-33 is elevated and promotes immune cell activation, leading to the development of asthma. However, which immune cells are responsible for triggering airway hyperreactivity (AHR), inflammation and eosinophilia remained to be clarified. We aimed to elucidate the individual roles of IL-33-activated innate immune cells, including ILC2s and $\mathrm{ST}^{+}$myeloid cells, in RSV infection-triggered pathophysiology.

Methods: The role of IL-33/ILC2 axis in RSV-induced AHR inflammation and eosinophilia were evaluated in the IL-33-deficient and YetCre-13 Rosa-DTA mice. Myeloidspecific, IL-33-deficient or ST2-deficient mice were employed to examine the role of IL-33 and ST2 signaling in myeloid cells.
\end{abstract}

Results: We found that IL-33-activated ILC2s were crucial for the development of AHR and airway inflammation, during RSV infection. ILC2-derived IL-13 was sufficient for RSV-driven AHR, since reconstitution of wild-type ILC2 rescued RSV-driven AHR in IL-13-deficient mice. Meanwhile, myeloid cell-derived IL-33 was required for airway inflammation, $\mathrm{ST}^{+}$myeloid cells contributed to exacerbation of airway inflammation, suggesting the importance of IL-33 signaling in these cells. Local and peripheral eosinophilia is linked to both ILC2 and myeloid IL-33 signaling.

Conclusions: This study highlights the importance of IL-33-activated ILC2s in mediating RSV-triggered AHR and eosinophilia. In addition, IL-33 signaling in myeloid cells is crucial for airway inflammation.

KEYWORDS

asthma, eosinophilia, IL-33, ILC2, respiratory syncytial virus 


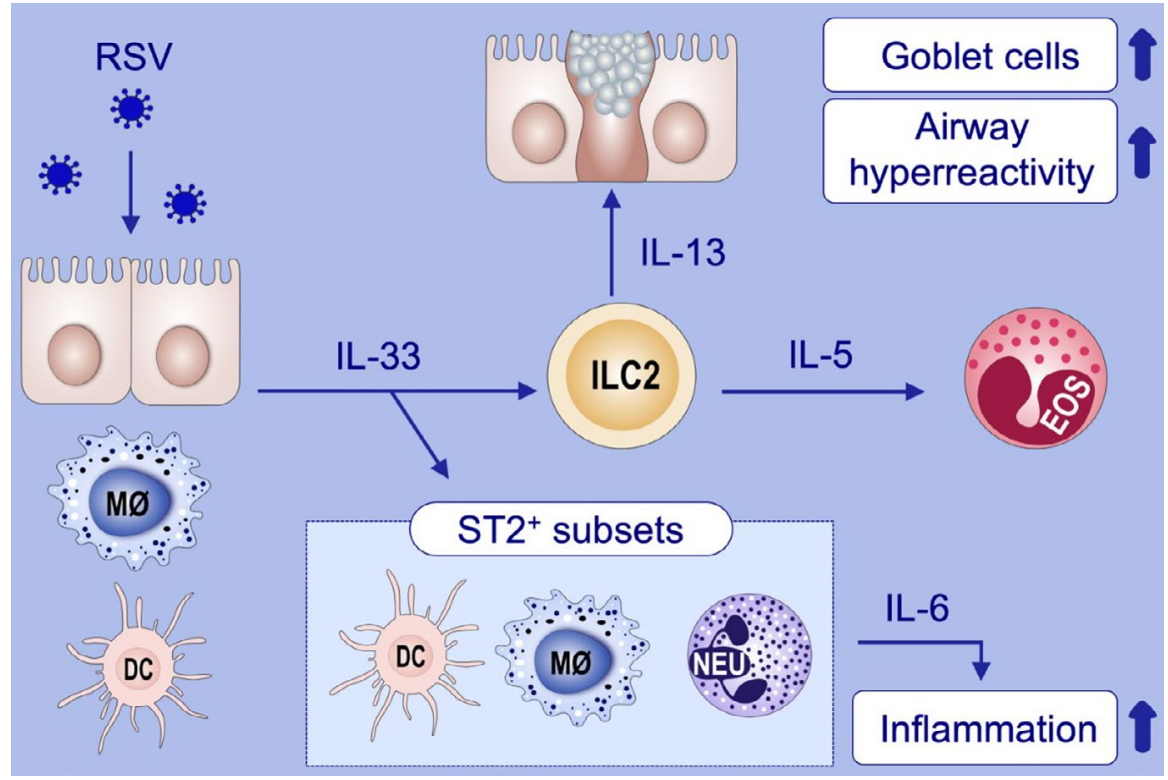

\section{GRAPHICAL ABSTRACT}

Respiratory syncytial virus induces ILC2 to produce IL-5 and IL-13 through IL-33, which is crucial for the development of airway hyperreactivity and airway inflammation. Myeloid cell-derived IL-33 and suppression of tumorigenicity 2-positive myeloid cells contribute to cytokine production and cellular inflammation in airway. Both ILC2 and myeloid cell IL-33 signaling contribute to local and peripheral eosinophilia.

\section{1 | INTRODUCTION}

Respiratory syncytial virus (RSV) causes lower respiratory tract infection and breathlessness, leading to the hospitalization of infants and immunocompromised adults. ${ }^{1,2}$ The wheeziness pertaining to RSV infection has been correlated to the predisposition of atopy. ${ }^{3}$ Subjects with RSV bronchiolitis in their infancy are more likely to develop asthma and atopic symptoms in the early adulthood. ${ }^{4}$ Immunologically, greater numbers of polymorphonuclear cells and lymphocytes and elevated levels of leukotrienes and prostaglandins have been observed. ${ }^{1}$ RSV infection also induces a wide spectrum of pro-inflammatory cytokines such as IL-6, 5,6 which have been suggested to contribute to the pathogenesis of RSV-induced bronchiolitis. $^{7,8}$

In murine models, early-life RSV infection leads to sensitization against multiple allergens and development of type 2 immune responses, including acute eosinophilia in the lungs. ${ }^{9-11}$ Th2 cytokines, such as IL-5 and IL-13, are elevated in the bronchoalveolar lavage fluid (BALF) of RSV-infected children, ${ }^{12,13}$ suggesting a role for these cytokines in RSV pathogenesis. Notably, RSV strains such as $A 2^{14}$ and $\mathrm{L} 19^{15}$ can boost Th2 cytokines, mucus production or AHR without the need of allergen predisposition. On the other hand, long strain can boost AHR and eosinophilia but not Th2 cytokine production in the absence of allergen predisposition. ${ }^{11}$ Interestingly, A2 strain exacerbates AHR in mice when infected after OVA sensitization, but suppresses AHR and lung eosinophilia when the infection precedes OVA sensitization. ${ }^{16}$ Since AHR typically develops within the first week after RSV infection in long and L19 strains, ${ }^{11,15}$ the innate immunity likely contributes to the initiation of airway inflammation during the acute phase. ${ }^{17}$

Innate lymphoid cells (ILCs) are a group of non-B, non-T lymphocytes that do not undergo antigen receptor rearrangement during their development. Group 2 innate lymphoid cells (ILC2s) are a member of the ILC family, which require GATA3 for their development and function ${ }^{18}$ and play prominent roles in helminth expulsion, ${ }^{19}$ airway inflammation, ${ }^{20}$ and atopic dermatitis. ${ }^{21}$ ILC2s are activated by epithelial-derived cytokines, IL-25, IL-33, and thymic stromal lymphopoietin (TSLP) through their cognate receptors, IL17RB, ST2, and TSLPR, respectively, and produce copious amounts of the Th2 cytokines IL-13 and IL-5. ${ }^{22}$ Previous study has demonstrated that viral infection expands ILC2 population ${ }^{23}$ and that they are the major source of IL-5 ${ }^{24}$ and IL-13 ${ }^{25}$ contributing to the development of eosinophilia and AHR, respectively.

IL-33 is a nuclear cytokine that is constitutively expressed in structural cells like type 2 pneumocytes in mice and lung epithelial cells in humans ${ }^{26}$ and can be induced in hematopoietic cells. ${ }^{27}$ IL-33 is released from cell upon tissue damage and binds to its receptor, ST2. ${ }^{26}$ Yet, the role of IL-33 in RSV infection remains controversial. Qi et $\mathrm{al}^{28}$ demonstrated that IL-33 plays a pivotal role in RSV-driven airway inflammation in mice. However, Stier et al found IL-33 to be dispensable for AHR onset and IL-13 production by ILC2 during infection. ${ }^{29}$ Moreover, although myeloid cell lines have been shown to produce IL-33 after infection, ${ }^{30}$ the physiological relevance of myeloid-derived IL-33 is still undefined.

In current study, we investigated the role of myeloid-derived IL-33 in the acute phase of RSV-driven airway inflammation. First, 
we found that ILC2 expansion, AHR, and subsequent eosinophilia are mediated by IL-33. We also demonstrated that myeloid-derived IL-33 and myeloid-dependent ST2 signaling are required for RSV-driven neutrophilic infiltration and IL-6 production. Although myeloid-specific depletion of IL-33 or ST2 did not affect AHR and IL-13 level, IL-5 and eosinophilia were markedly reduced. Overall, this study offers new insight into the regulatory roles of IL-33 and its downstream innate cells during the acute phase of RSV-induced AHR, airway inflammation, and peripheral eosinophilia.

\section{2 | MATERIALS AND METHODS}

Please refer to the Appendix S1 for further methodological details.

\section{1 | RSV infection}

Human RSV strain line 19 (L19) was propagated in Hep-2 cells maintained in Eagle's MEM containing 5\% heat-inactivated FBS as previously described. ${ }^{15}$ Mice were inoculated under light anesthesia (isoflurane) by intranasal instillation of $10^{6} \mathrm{PFU}$ of purified virus in $75 \mu \mathrm{L}$ endotoxin-free PBS unless otherwise stated. Sham-infected animals were inoculated with lysed HEp2 cells under identical conditions.

\subsection{Measurement of airway responsiveness (AHR) in the mouse model}

Mice were anesthetized with $100 \mathrm{mg} / \mathrm{kg}$ pentobarbital. Mice were tracheostomized, intubated, and mechanically ventilated at a tidal volume of $0.2 \mathrm{~mL}$ and a frequency of 150 breath/min, as previously described. ${ }^{31}$ Lung function was determined by measuring airway resistance $\left(R_{L}\right)$ in response to increasing doses $(0.125$ to $40 \mathrm{mg} / \mathrm{mL})$ of aerosolized acetyl- $\beta$-methylcholine chloride (Sigma-Aldrich) via the FinePointe RC system (Buxco Research Systems).

\subsection{Collection and analysis of bronchoalveolar lavage fluid}

Mice were euthanized and the lungs were lavaged twice with $0.5 \mathrm{~mL}$ of PBS, and the fluid was pooled. Cells were spun onto glass slides by CYTO-TEK ${ }^{\circledR}$ Cytocentrifuge and stained with Diff-Quick solution (Sysmex). Cells in BALF were counted and analyzed, as previously described. ${ }^{32}$

\section{4 | Flow cytometry}

Single cell suspensions were preincubated with Fixable Viability Dye and anti-Fc $\gamma$ blocking mAb (2.4G2) and then washed before staining with surface antibodies. For intracellular staining, single cell suspensions were incubated with $5 \mathrm{ng} / \mathrm{mL}$ phorbol 12-myristate 13-acetate (PMA), $500 \mathrm{ng} / \mathrm{mL}$ ionomycin, and $2 \mu \mathrm{mol} / \mathrm{L}$ Golgi stop A (BD Biosciences) for 6 hours prior to surface staining. After surface staining, cells were fixed and permeabilized with Cytofix/Cytoperm solution (BD Biosciences), and further stained intracellularly with the appropriate antibodies. Flow cytometry was performed on a LSRII flow cytometer (BD Biosciences), and data were analyzed using FlowJo 10 software (Tree Star, Inc). Refer to Table S1 for the full list of flow cytometry antibodies used in the study.

\section{5 | RT-qPCR and ELISA}

Refer to Appendix S1 for details. See Table S2 for the list of primers used for qPCR in lung samples.

\subsection{Adoptive transfer of ILC2}

Lung ILC2s $\left(\mathrm{CD}_{4} 5^{+}\right.$Lineage $\left.\mathrm{ST}^{+}\right)$were sorted from donor mice receiving IL-33 $(1 \mu \mathrm{g})$ intranasally and sacrificed five days later. Sorted lung ILC2s were adoptively transferred to $1 / 13^{-/-}$recipients $\left(10^{5}\right.$ cells/ mouse) through the intratracheal route 1 hour prior to RSV infection.

\section{7 | Statistical tests}

All data were analyzed using Prism 6 (GraphPad Software Inc) and presented as means \pm SEM. Statistical significance between groups was determined by two-way ANOVA or unpaired student's $t$ tests (two-tailed) unless specified otherwise, where $P<.05$ was considered significant.

\section{3 | RESULTS}

\section{1 | RSV infection prompts the onset of IL-33- dependent AHR and mucus production}

To recapitulate RSV-driven wheezing illness, mice were infected with RSV (L19) and the resulting airway resistance was measured 6 days post-infection (d.p.i.). We showed that RSV infection-triggered AHR in mice (Figure 1A). To determine the extent of leukocyte infiltration, BALF cell content was analyzed. BALF neutrophil infiltration preceded eosinophil accumulation and was observed as early as day 6 post-infection. Eosinophils, on the other hand, emerged on day 9 (Figure 1B). To ascertain the presence of eosinophils in the lungs following RSV infection, we performed flow cytometry and observed that similar to BALF, lung eosinophil numbers increased on day 9 (Figure 1C,D). Meanwhile, the mRNA level of Gob5, a mucus-associated gene, was greatly increased in RSV-infected lungs (Figure 1E). The results were consistent with previous findings. ${ }^{15,33}$ Taken together, the model of RSV-driven airway resistance was established.

Previous evidence suggests that IL-33 can be produced in the lungs upon RSV infection, both in adults ${ }^{29}$ and in neonatal mice, ${ }^{34}$ but the necessity of IL-33 in adult mice has not been addressed. We found that IL-33 in BALF peaked on day 3 following RSV infection and slightly declined by 6 d.p.i. in adult mice (Figure 1F). We further examined the role of IL-33 in RSV-induced airway inflammation in adult $1 / 33^{-/-}$mice. In comparison with wild-type (WT) mice, 

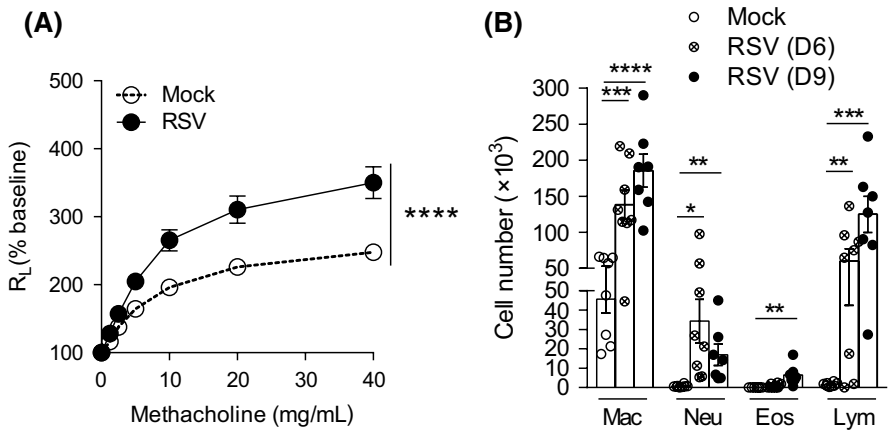

(C)

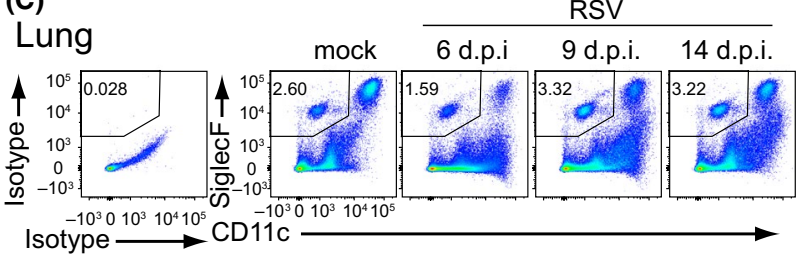

(D)
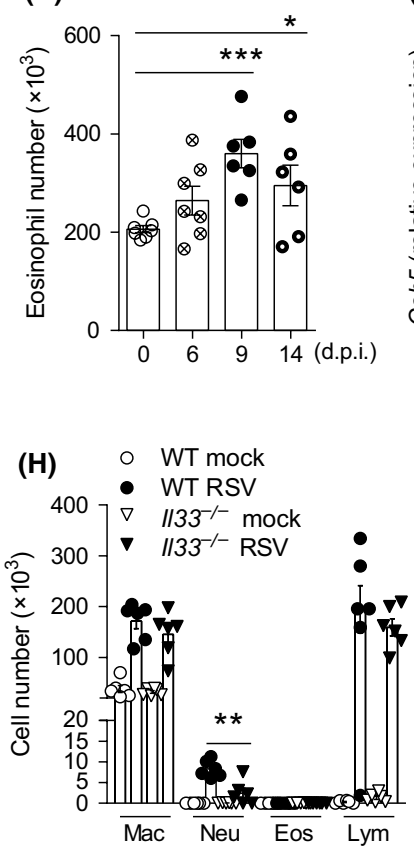

(E)

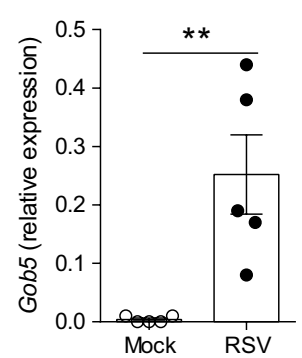

(I)

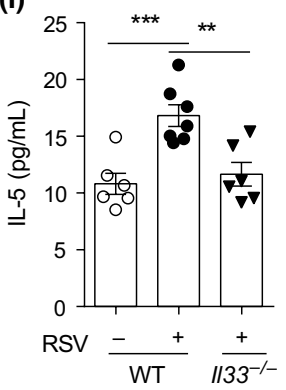

(F)

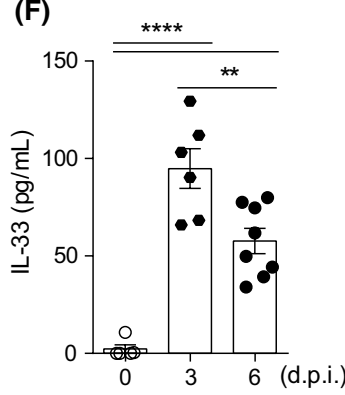

(G) $\quad \odot$ WT Mock

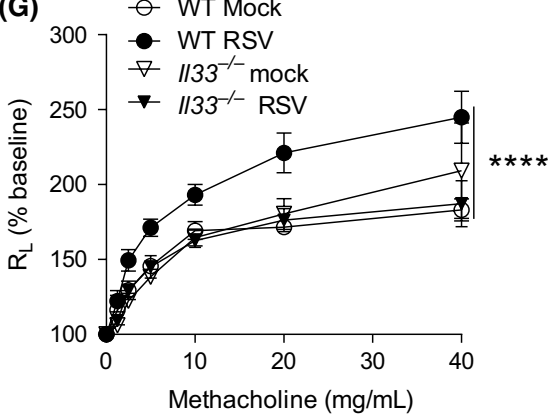

FIGURE 1 Respiratory syncytial virus (RSV) infection induces airway hyperreactivity (AHR) and mucus production in an interleukin (IL)-33-dependent manner. (A) Changes in lung resistance $\left(R_{L}\right)$ of BALB/c mice infected with RSV line 19 (L19) (106 PFU/mouse) or mock and sacrificed on day 6 post-infection (d.p.i.). $n=7-9$. (B) Cellular composition in the bronchoalveolar lavage fluid (BALF) of BALB/c mice on day 6 or day 9 post-infection. $n=7-8$. (C-D) BALB/c mice were infected with RSV L19 (10 PFU/mouse) and sacrificed on indicated time point. (C) Representative flow cytometry plot showing lung eosinophils (CD45 ${ }^{+}$CD $11 c^{-}$SiglecF $F^{+}$), assessed by FACS. (D) Total numbers of lung eosinophils. $n=6-7$. (E) Gob5 mRNA expression in the lungs of BALB/c mice infected with RSV L19 or mock, analyzed by RT-qPCR on 6 d.p.i. $n=5$. (F) IL-33 in BALF from BALB/c mice under RSV infection at indicated time points. $n=5-8(G-K) ~ I I 33^{-/-}$and WT littermates were infected with RSV L19 and sacrificed 6 d.p.i.. (G) Changes in lung resistance $\left(R_{L}\right) . n=6$. (H) Cellular composition in the bronchoalveolar lavage fluid (BALF) $n=6$. (I) IL-5 in BALF (left) and lung (right) and (J) IL-13 level in lung homogenates, assessed by ELISA. (K) Gob5 mRNA expression in the lungs, quantified by RT-qPCR. $n=6-8$. d.p.i, days post-infection; Eos, eosinophil; IL, interleukin; Lym, lymphocyte; Mac, macrophage; Neu, neutrophil. Data were pooled from 2 independent experiments. ${ }^{*} P<.05,{ }^{* *} P<.01,{ }^{* * *} P<.001$, and ${ }^{* * * *} P<.0001$

FIGURE 2 Respiratory syncytial virus (RSV) infection induces group 2 innate lymphoid cell (ILC2) activity in lungs. (A-B) BALB/c mice were infected with RSV L19 (106 PFU) or mock and sacrificed 6 d post-infection (d.p.i.). (A) Representative flow diagram showing gating strategy of thymus cell antigen 1.2 (Thy1.2) and GATA binding protein 3 (GATA3) expression in lung ILC2s (CD45 $^{+}$Lineage $^{-} \mathrm{ST}^{+}{ }^{+}$c-Kit ${ }^{+}$ Sca- $1^{+}$). (B) Absolute numbers of ILC2s in the lungs of mice after infection. $n=5-7$. (C-D) BALB/c mice were infected with RSV L19 (10 ${ }^{6}$ PFU) or mock and analyzed for IL-5 and IL-13 expression by FACS. (C) Gating strategy of IL- $5^{+}$and IL-13 $3^{+}$ILC2s $_{\text {(CD } 45^{+} \text {Lineage }^{-} \text {ST2 } 2^{+} \text {C-Kit }}{ }^{+}$ Sca- $1^{+}$). (D) Total numbers of IL-5 $5^{+}$and IL-13 $3^{+}$ILC2s. $n=5-7$. (E-G) $1133^{-/-}$and WT littermates were infected with RSV L19 (10 ${ }^{6}$ PFU) or mock and analyzed for IL-5 and IL-13 expression in ILC2s (CD45 ${ }^{+}$Lineage ${ }^{-}$ST2 ${ }^{+}$c- Kit $^{+}$Sca- ${ }^{+}$) by FACS. (E) Gating strategy, (F) total numbers of ILC2 (CD45 ${ }^{+}$Lineage ${ }^{-}$ST2 ${ }^{+}$c-Kit ${ }^{+}$Sca- $\left.1^{+}\right)$, and (G) total number of IL- $5^{+}$and IL-13 ${ }^{+}$ILC2s. $n=9$. c-kit, proto-oncogene tyrosine-protein kinase Kit; FSC, forward scatter; FVD, fixable viability dye; Sca-1, stem cells antigen-1; SSC, side scatter; ST2, suppression of tumorigenicity 2. Data were pooled from 2 independent experiments. ${ }^{*} P<.05,{ }^{* *} P<.01$ and ${ }^{* * * *} P<.0001$ 
(A)

Lung
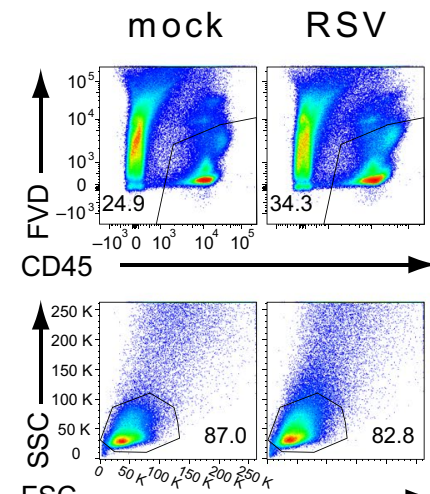

FSC ${ }^{5} 0_{k}^{100} k^{150} k^{200} k_{k}^{250}$

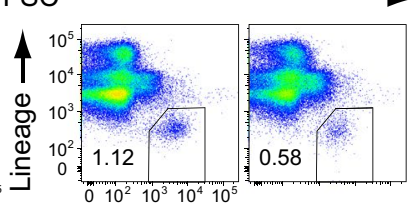

Isotype $\stackrel{0}{\longrightarrow} \stackrel{10^{2} 10^{3} 10^{4}}{\longrightarrow}$
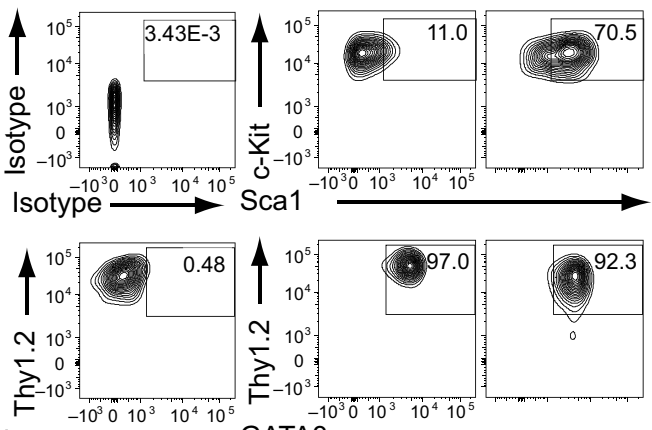
Isotype

(E)

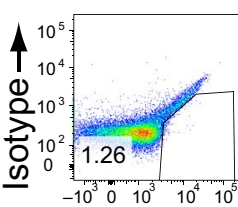

Isotype $\longrightarrow$

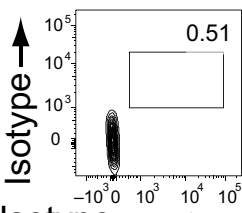

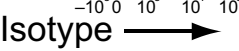

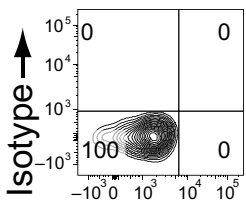

Isotype
GATA3
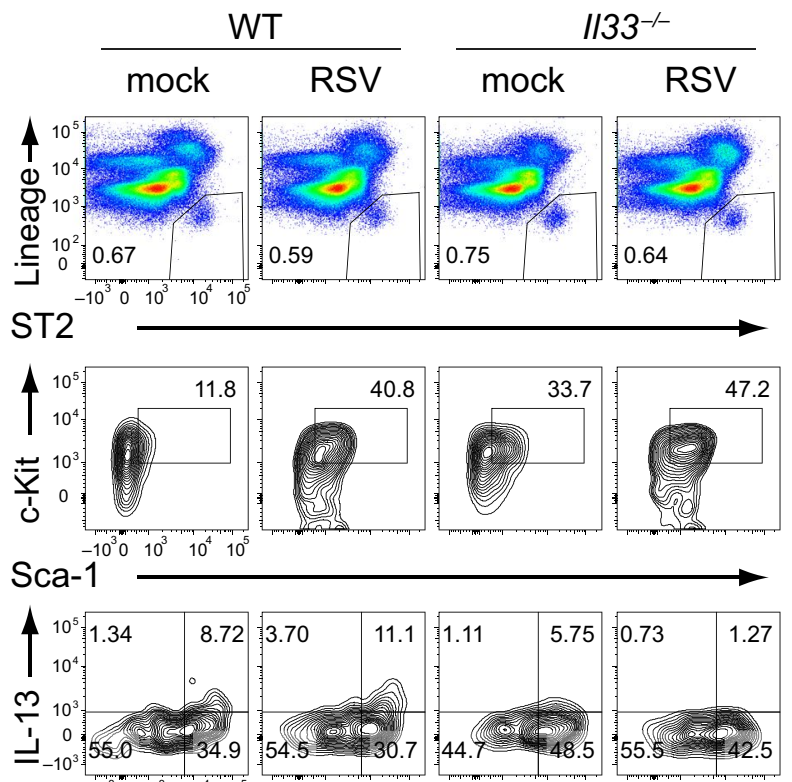

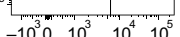

IL-5
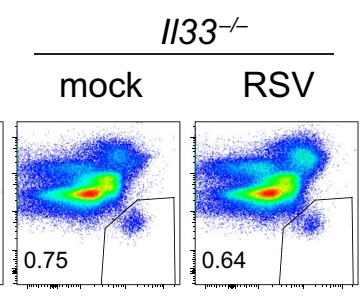

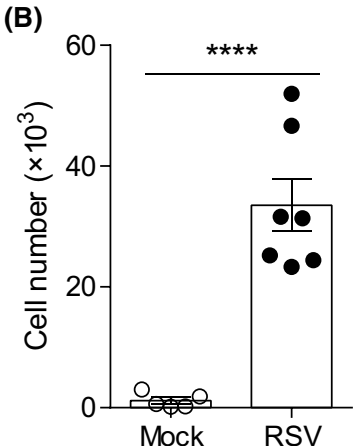

(C)
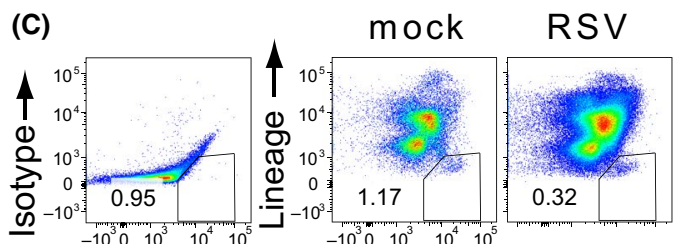

Isotype $\stackrel{-10^{3}}{\longrightarrow} \quad 10^{4} \quad 10^{5}$
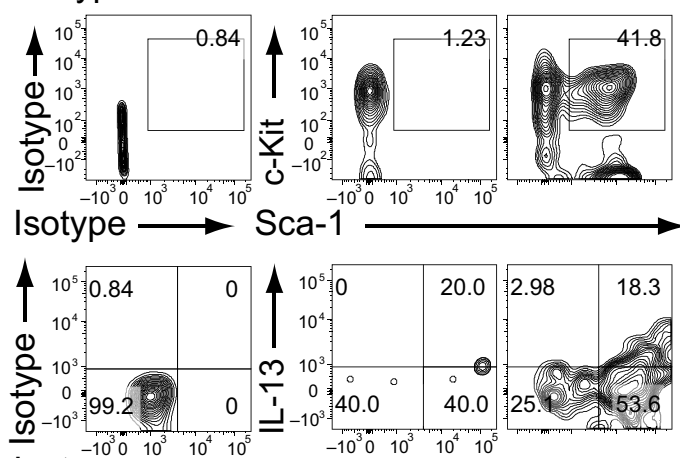

Isotype

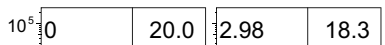

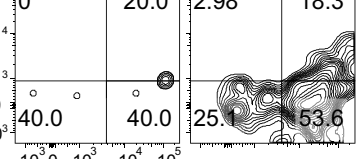

$-10^{3} 0$\begin{tabular}{ccc}
$10^{3}$ & $10^{4}$ & $10^{5}$ \\
\hline
\end{tabular}

(D)

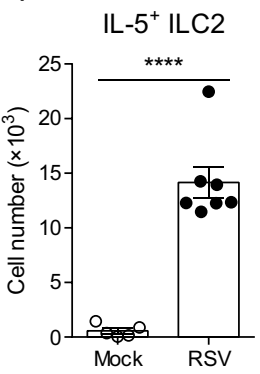

IL-13 ${ }^{+}$ILC2

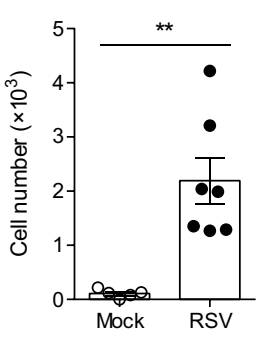

(G)

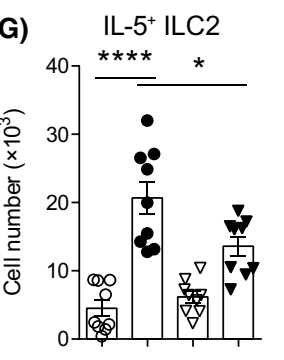

RSV

WT $\frac{-}{1133^{-1}}$
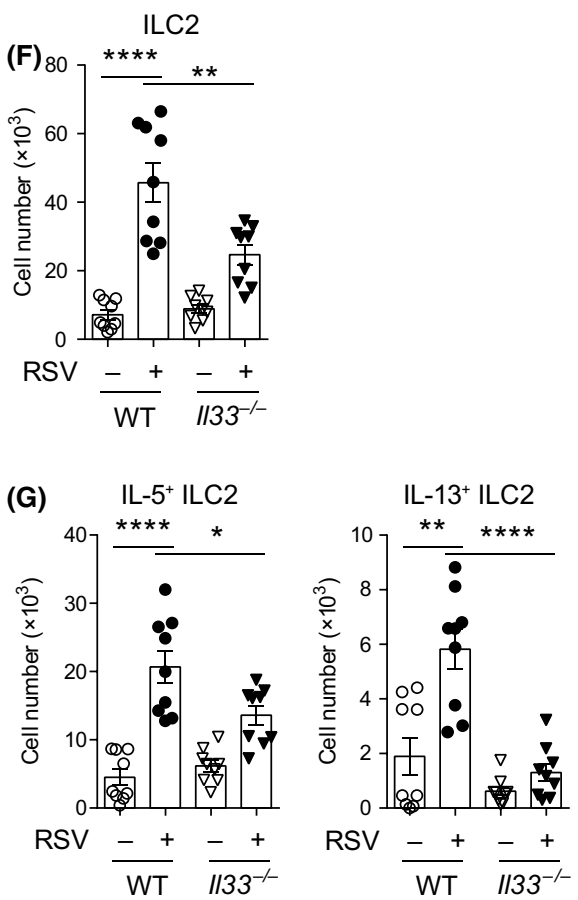

$1 / 33^{-/-}$mice developed lower AHR (Figure 1G) and neutrophil infiltration in BALF (Figure 1H). Also, we found that IL-33 deficiency attenuated IL-5 and IL-13 production (Figure 1I,J), and Gob5 expression
(Figure $1 \mathrm{~K}$ ) in response to RSV infection. Overall, our results demonstrated that IL-33 is crucial for RSV-driven airway inflammation in adult mice. 
(A)

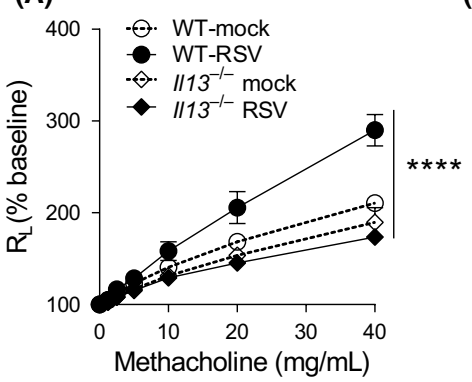

(E)

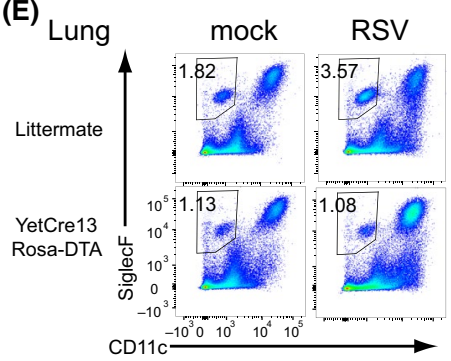

(H)

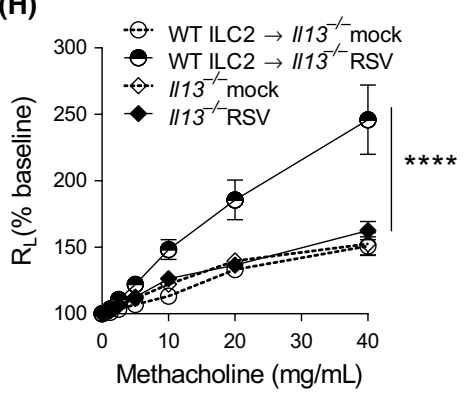

(B)

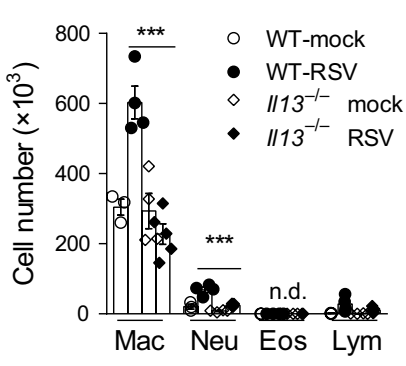

(C)

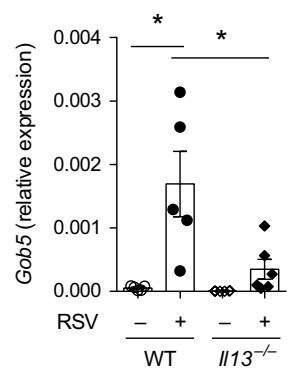

(D)

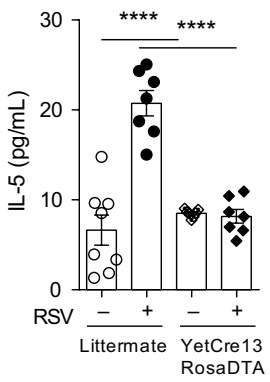

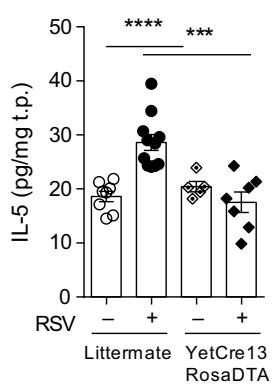

(F)

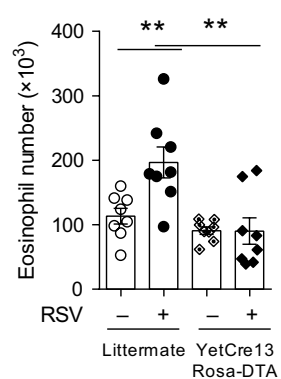

(G)
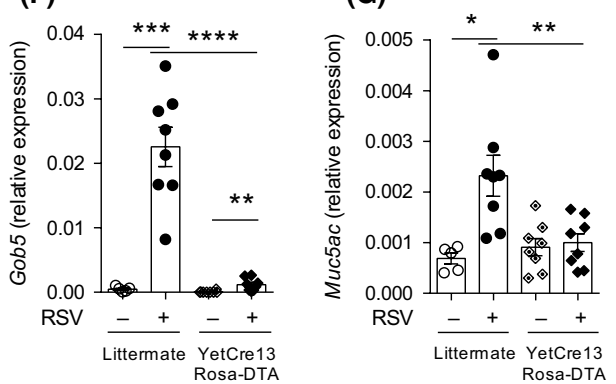

(I)

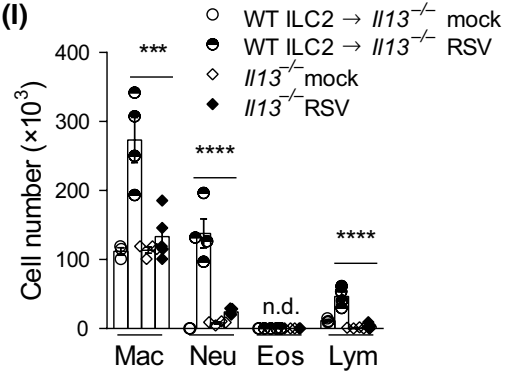

FI G URE 3 ILC2-derived IL-13 is sufficient for respiratory syncytial virus (RSV)-driven AHR, lung inflammation, and eosinophilia. (A-B) $1113^{-/-}$and wild-type (WT) mice were infected with RSV or mock and sacrificed $6 \mathrm{~d}$ post-infection (d.p.i.). (A) Changes in lung resistance $\left(R_{\mathrm{L}}\right)$ $n=5-8$. (B) Cellular composition in the BALF of mice after infection. $n=3-5$. (C) mRNA levels of Gob5 in the lungs of $1113^{-1-}$ and WT mice infected with RSV. $n=5-6$. (D-G) YetCre-13 Rosa-DTA and WT littermates were infected with RSV or mock and sacrificed. (D) IL-5 in BALF (left) and lung homogenate (right) were determined on 6 d.p.i. $n=5-8$. (E) Representative flow cytometry plot (left panel) and total numbers (right panel) of lung eosinophils (CD45 ${ }^{+} \mathrm{CD}_{11 c^{-}}$SiglecF $\mathrm{F}^{+}$), assessed by FACS on 9 d.p.i. $\mathrm{n}=5-8$. (F-G) Levels of $\mathrm{Gob5}$ and Muc5ac mRNA

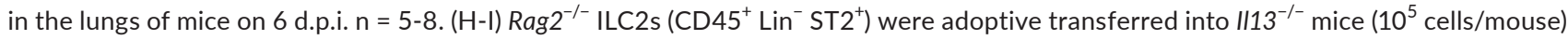
intratracheally, followed by RSV or mock infection. Mice were sacrificed $6 \mathrm{~d}$ post-infection. $(H)$ Changes in lung resistance $\left(R_{\mathrm{L}}\right) \cdot n=3-4$. (I) Cellular composition in the BALF. $n=3-5$. Eos, eosinophil; Lym, lymphocyte; Mac, macrophage; n.d., not detectable; Neu, neutrophil. Data were pooled from 2 independent experiments. ${ }^{*} P<.05,{ }^{* *} P<.01,{ }^{* * *} P<.001$, and ${ }^{* * * *} P<.0001$

\subsection{RSV infection-initiated ILC2 expansion is IL-33-dependent}

To substantiate the role of IL-33 in ILC2 activation in the lungs, we isolated murine lung ILC2s (CD45 ${ }^{+}$Lineage $^{-} \mathrm{ST} 2^{+}$) (Figure S1A) and stimulated them with IL-33 in vitro. As expected, IL-33 induced IL-5 and IL-13 production by ILC2s at the mRNA (Figure S1B) and protein (Figure S1C,D) levels. Also, Sca-1 expression can be found in IL-33-activated ILC2 (Figure S1E). To ascertain that IL-33 signaling is ST2-dependent in lung ILC2s, we treated $\mathrm{St}^{-/-}$mice with IL-33. No significant differences in the percentages (Figure S1F) and total numbers (Figure S1G) of lung ILC2s were observed between mock- and IL-33-treated St2 ${ }^{-/-}$mice. In short, IL-33 can induce ILC2s to express IL-5 and IL-13 in the lungs in an ST2-dependent manner.

To test if RSV infection can expand pulmonary ILC2s, we analyzed ILC2 population ( $\mathrm{CD} 45^{+}$Lineage $\left.{ }^{-} \mathrm{ST} 2^{+} \mathrm{Sca} 1^{+} \mathrm{C}-\mathrm{Kit}^{+}\right)$in the lungs by flow cytometry 6 d.p.i. The expression of both GATA3 and Thy 1.2 from ILC2 agrees with previous studies (Figure 2A). ${ }^{18}$ RSV infection increased the frequencies and number of ILC2s in lungs (Figure 2A,B). Also, both IL-5 and IL-13 production were increased in ILC2s after RSV infection, assessed by intracellular staining (Figure 2C,D). Notably, the ILC2 accumulation and the induction of IL-5 and IL-13 in ILC2s required IL-33, given that total ILC2 as well as IL- $5^{+}$and IL-13 ${ }^{+} \mathrm{ILC} 2 \mathrm{~s}$ were reduced in $1133^{-/-}$mice comparing to WT after RSV infection (Figure 2E-G). 


\section{3 | ILC2-derived IL-13 is sufficient for RSV-driven AHR, airway inflammation, and eosinophilia}

Although several studies have demonstrated the importance of IL-13 in RSV-driven airway inflammation, the role of ST2 ILC2s in RSVdriven AHR is still uncertain. ${ }^{14,29}$ Thus, we planned to determine whether IL-13 expression by ILC2s is sufficient to cause symptoms associated with RSV infection. First, to prove that RSV-induced airway resistance and inflammation are mediated by IL-13, we measured AHR, BALF cellularity, and expression of Gob5 in $1113^{-/-}$mice. IL-13-deficient mice showed reduced AHR (Figure 3A), BALF leukocyte infiltration (Figure 3B), and Gob5 expression (Figure 3C) on 6 d.p.i. To clarify the involvement of ILC2s, we used the YetCre-13 mT/ $\mathrm{mG}$ mice, which provides fate-mapping of IL-13-positive cells under RSV infection. We showed that ILC2 is the major cellular source of IL-13 in the lungs (Figure S2A). Accordingly, the percentage of IL-13-producing ILC2s increased after RSV infection (Figure S2B). Although mast cells also produced certain level of IL-13 in mocktreated mice, RSV infection did not further increase its production. Meanwhile, CD4 T cells and basophils did not produce IL-13 after RSV infection. (Figure S2B). Additionally, we used the YetCre-13 Rosa-DTA mice, which selectively depletes IL-13-producing cells. ${ }^{35}$ Upon IL-33 treatment, the population of lung ILC2s were markedly lower in YetCre-13 Rosa-DTA mice compared to their WT littermates (Figure S2C). Meanwhile, RSV-infected YetCre-13 Rosa-DTA mice showed diminished lung IL-5 and eosinophilia on day 6 and 9 postinfection, respectively, (Figure 3D,E), as well as reduced Gob5 and Muc5ac mRNA levels in the lungs (Figure 3F,G). To prove that ILC2derived IL-13 is sufficient to drive AHR under RSV infection, we isolated IL-33-stimulated ST2 ${ }^{+}$ILC2s from Rag $2^{-/-}$mice and adoptively transferred these lymphocytes into the lungs of $1113^{-/-}$mice. We found that WT ILC2 reconstitution restored both AHR (Figure $3 \mathrm{H}$ ) and leukocyte infiltration (Figure 3I) driven by RSV infection.

\subsection{Myeloid cell-derived IL-33 contributes to the onset of airway inflammation in the lungs in an ST2- dependent manner}

To verify the expression profile of IL-33 under RSV infection, we performed CD11b and IL-33 co-staining in lung tissue sections. We found co-localization of IL-33 with both CD11 ${ }^{+}$myeloid cells and SP-C $\mathrm{C}^{+}$type- 2 pneumocytes. Both $\mathrm{CD} 11 \mathrm{~b}^{+}$and $\mathrm{SP}-\mathrm{C}^{+}$cells showed greater IL-33 expression after RSV infection (Figure 4A,B). Notably, total IL-33 ${ }^{+}$cells also increased after infection (Figure 4B). To uncover the source of IL-33 during the initial stage of RSV infection, we analyzed the cellular profile of IL-33 expression in the lungs on day 1 post-infection using flow cytometry. Our results showed that IL-33 expression in airway epithelial cells (CD45- EpCAM $\left.^{+} \mathrm{CD}^{-} 1^{-}\right)$ was induced after RSV infection (Figure 4C,D). Additionally, IL-33expressing lung myeloid cells, including alveolar macrophages (AM), interstitial macrophages (IM), and dendritic cells (DC), increased in both percentages (Figure 4C,E) and numbers (Figure 4E) after infection. To support the experimental observations above, we examined the mRNA level of IL-33 in BMDC and alveolar macrophage cell line $\mathrm{MH}-\mathrm{S}$ after RSV infection and observed similar induction in both cell types (Figure 4F,G).

Since myeloid cells are pivotal instigators of inflammation, we examined their role in RSV-induced inflammation by using myeloid cell-specific IL-33 knockout (II33 f/f LysM ${ }^{\text {Cre }}$ ) mice on 6 d.p.i.. Indeed, IL-33 expression in myeloid cells but not structural cells was abolished in $1133^{f / f}$ LysM $^{\text {cre }}$ mice (Figure S3A). The IL-33 protein level in BALF was also reduced in these mice (Figure $4 \mathrm{H}$ ). Although AHR response and II13 expression in the lungs of $/ 133^{f / f} L_{\text {LSM }}{ }^{\mathrm{Cre}}$ were induced to similar levels as their $1133^{f / f}$ littermates after RSV infection (Figure $4 \mathrm{I}, \mathrm{J}$ ), airway inflammation was attenuated, as evidenced by reduced neutrophil numbers in BALF (Figure $4 \mathrm{~K}$ ). To confirm that the observed reduction is not due to impaired neutrophil response, given that Lys ${ }^{\text {cre }}$ locus is also expressed in neutrophils, ${ }^{36}$ we treated these mice with IL-33-independent stimuli IL-1 $\beta$ and IL-23 and found that neutrophils responded similarly between $\mathrm{WT}, 1133^{-/}$, and $1133^{\mathrm{f} /}$ ${ }^{f}$ LysM ${ }^{\text {cre }}$ mice (Figure S3B).

Additionally, IL-5 in both BALF and lung was partially reduced in $1 / 33^{f / f}$ LysM $M^{\text {cre }}$ mice on 6 d.p.i. compared to $/ 133^{f / f}$ littermate (Figure $4 \mathrm{~L}$ ). Similar to IL-5, $1 / 33^{f / f}$ Lys $^{\text {cre }}$ had lower eosinophil numbers in the lungs on 9 d.p.i. compared to their littermates (Figure 4M). Notably, IL-33 is known to drive the production of IL-6, an inflammatory cytokine associated with asthma, in multiple cell types. ${ }^{37}$ We therefore examined whether myeloid-derived IL-33 is required for IL-6 production under RSV infection. Accordingly, both IL-6 mRNA and protein expression was reduced in $1133^{f / f}$ Lys $^{\text {cre }}$ mice (Figure $4 \mathrm{~N}, \mathrm{O}$ ). Likewise, the lung mRNA level of goblet cell marker Gob5 was reduced in $I 133^{f / f}$ Lys $^{C r e}$ mice (Figure 4P).

To investigate the importance of ST2 signaling on myeloid cells, we generated myeloid-specific ST2-deficient mice (St2/f Ly$\left.s M^{c r e}\right)$. The efficiency and specificity of knockout was confirmed by flow cytometry. As expected, ST2 expression was diminished in myeloid but not lymphoid cells (Figure S3C). Targeting ST2 in myeloid cells did not impair the level of AHR (Figure 5A), but partially suppressed airway inflammation in terms of reduced macrophage and neutrophil numbers in BALF (Figure 5B). Although the mRNA and protein levels of IL- 6 were reduced in these mice (Figure 5C,D), II13 (Figure 5E) and Gob5 (Figure 5F) levels were unaffected. Hence, myeloid cell-derived IL-33 contributes to cytokine production and cellular infiltration in the airway but not $A H R$, and $\mathrm{ST}^{+}$myeloid cells are required for the production of IL- 6 in response to RSV infection.

Next, we examined whether IL-33 expression is linked to TSLP expression, another cytokine previously reported to be important in RSV-driven airway inflammation. ${ }^{29}$ We found that the mRNA level of Tslp was not affected by global knockout (Figure S4A) or myeloid cell-specific knockout (Figure S4B) of IL-33. Of note, RSV replication was independent of IL-33 as no significant differences in the level of viral mRNA was observed between $1133^{-/-}, 1133^{f / f}$ Lys $^{\text {Cre }}$ mice, and their respective WT littermates after infection (Figure S4C,D). Taken together, IL-33 produced by lung myeloid cells contributes to cellular infiltration but not AHR under RSV infection. 
To correlate the occurrence of cell death to RSV infection, we performed TUNEL assay on lung tissue sections from mock- and RSV-infected mice. As expected, we observed an increase in DNA
(A)

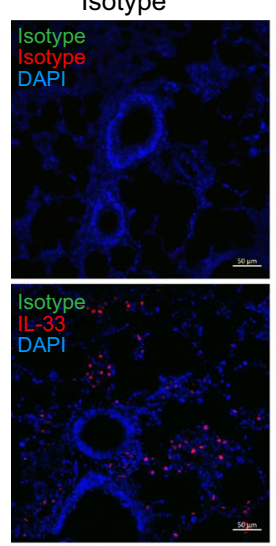

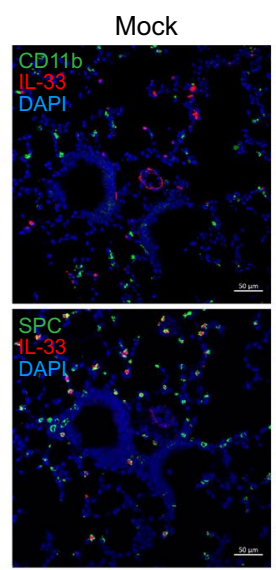

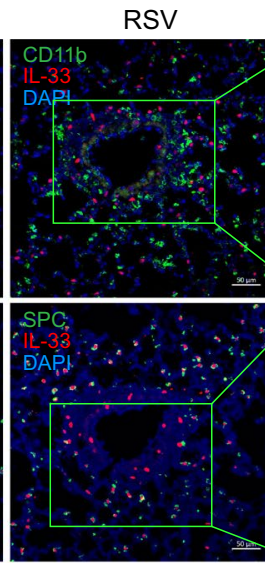
lung cells revealed increased frequencies of annexin $\mathrm{V}^{+} \mathrm{CD} 45^{-}$structural cells (Figure S5C) and CD45 ${ }^{+}$leukocytes (Figure S5D). These fragmentation after infection (Figure S5A,B). Annexin $V$ staining of

\section{(C)}

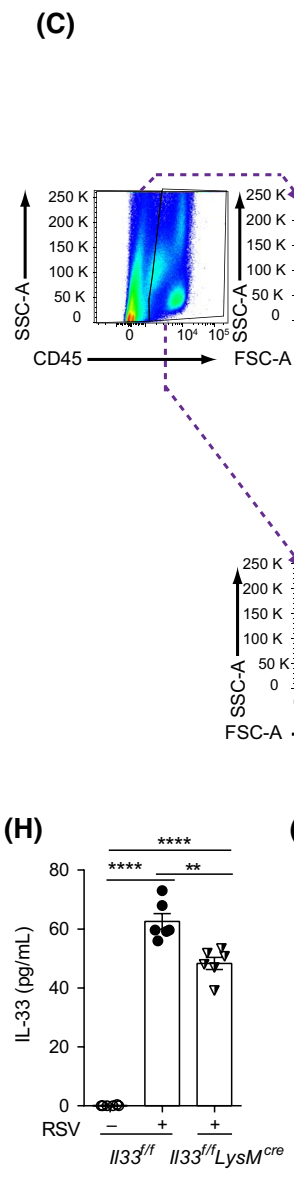

(1)
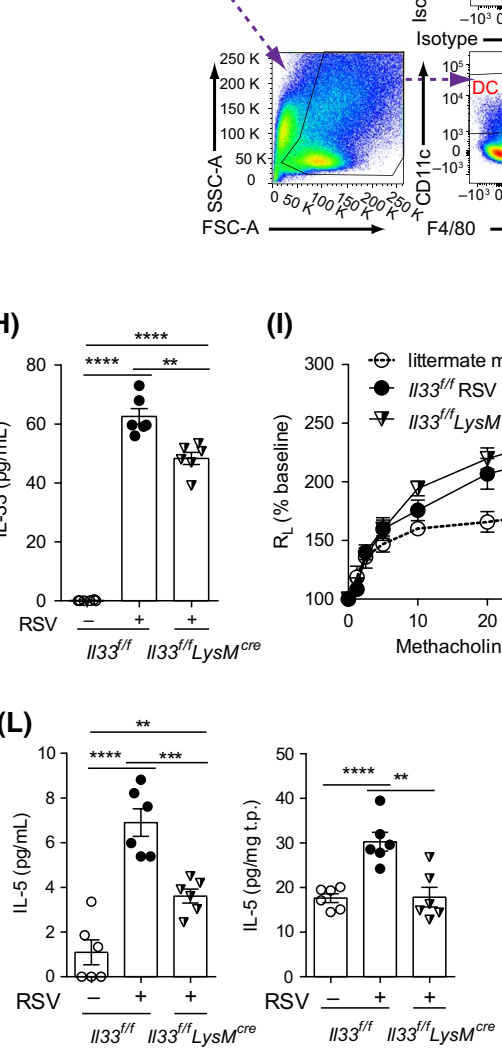
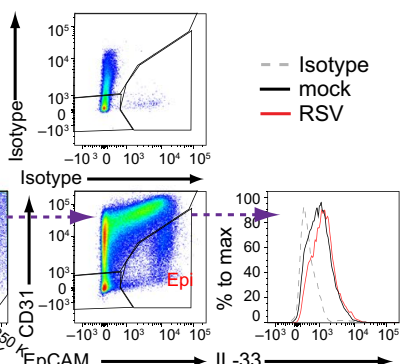

$\mathrm{IL}-33 \longrightarrow$

\begin{tabular}{llll} 
& 0 \\
\hline
\end{tabular}
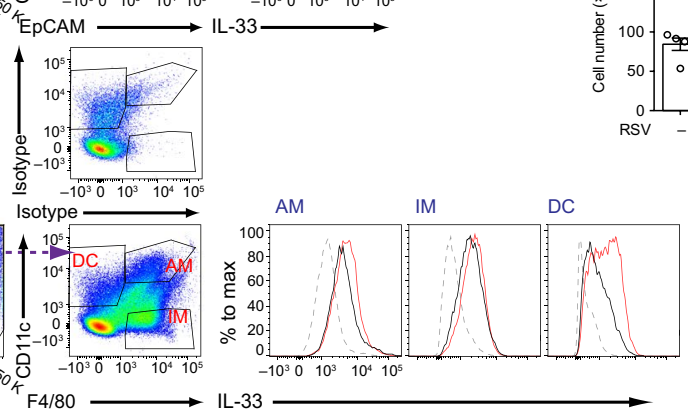

(B)

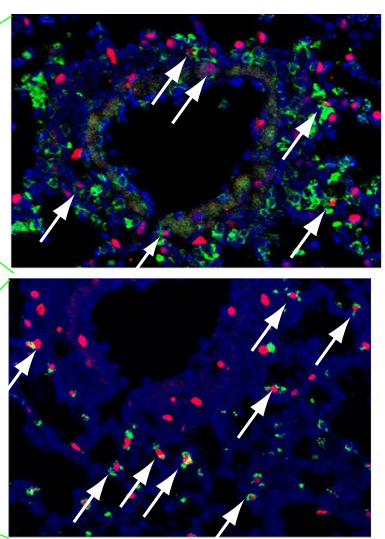

(1)

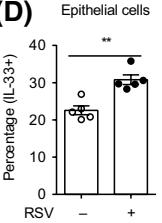

(E)

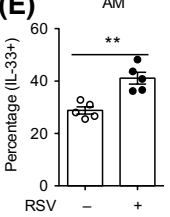

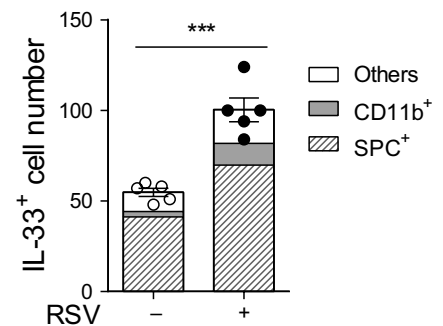
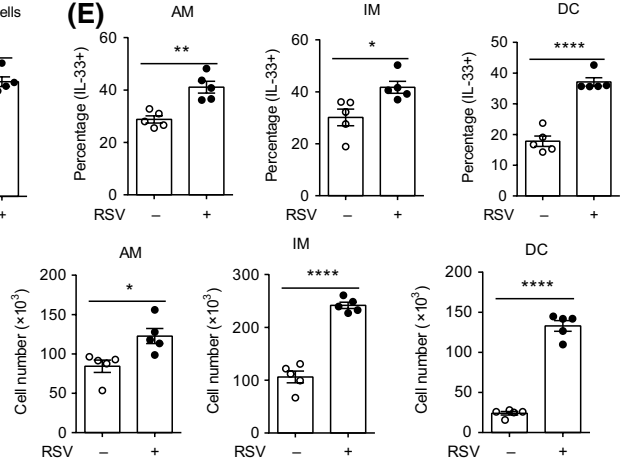

(F)
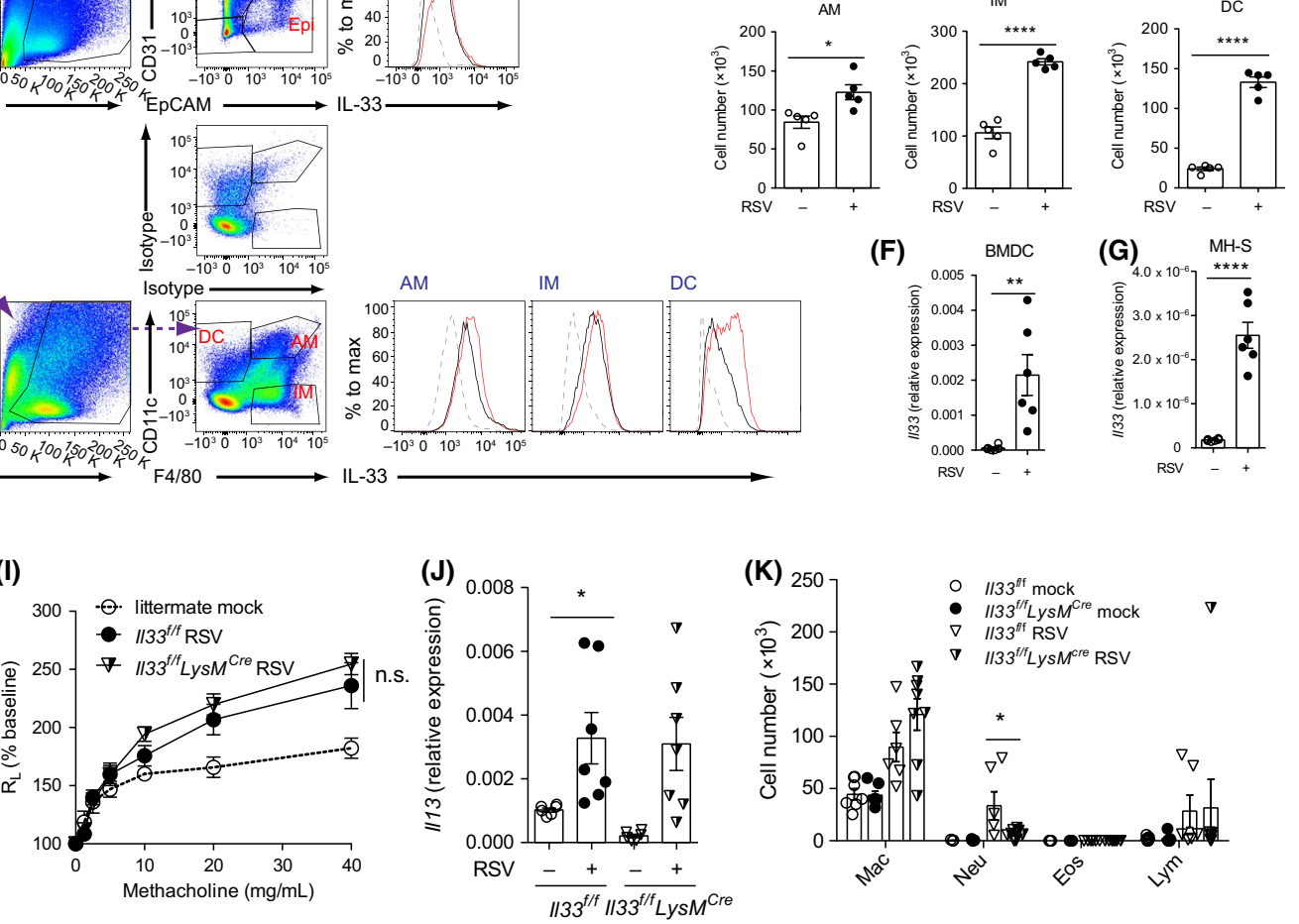

(M)

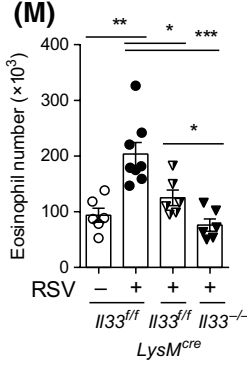

(N)

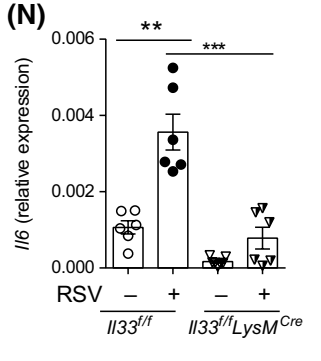

(0)

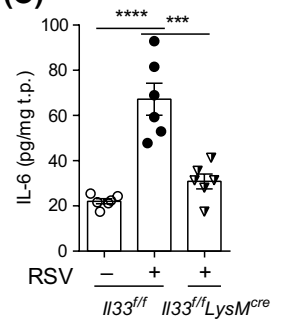

(P)

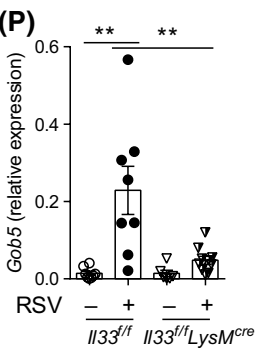


FIG URE 4 Myeloid-derived IL-33 contributes to RSV-induced airway inflammation, but not AHR. (A-B) Wild-type mice were infected with RSV L19 (106 PFU) or mock and sacrificed $1 \mathrm{~d}$ post-infection. Lung sections were subjected to CD11b, SP-C, and IL-33 immunofluorescence staining. Counter staining was performed using DAPI nuclear staining. (A) Representative immunofluorescence images.

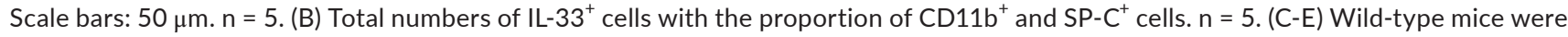
infected with RSV $\left(10^{6} \mathrm{PFU}\right)$ or mock and sacrificed 24 hours later. (C) Representative flow cytometry plot showing IL-33 ${ }^{+}$epithelial cells

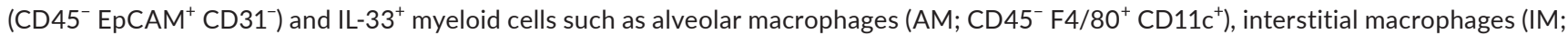

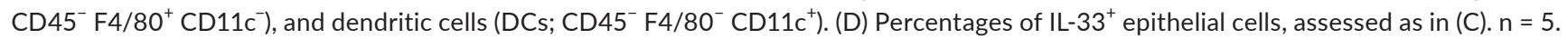
(E) Percentage (upper panel) and total number (lower panel) of IL-33+ AM, IM, and DCs, assessed as in (C). $n=5$. (F) Level of $I I 33 \mathrm{mRNA}$ in bone marrow-derived dendritic cells (BMDC) after 6-hour infection with RSV L19 (MOI = 1), n = 6. (G) Level of II33 mRNA in murine alveolar

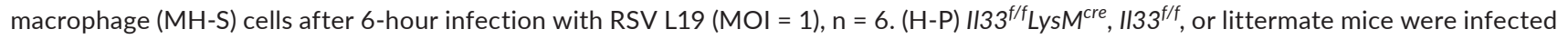
with RSV or mock and sacrificed $6 \mathrm{~d}$ post-infection (except mice in (L) were sacrificed on 9 d.p.i.). (H) IL-33 in BALF were determined by ELISA, $n=6$. (I) Changes in lung resistance $\left(R_{L}\right), n=5-6$. (J) $I 113$ in mRNA in lung was determined by $q P C R, n=6-7$ (K) Cellular composition in the BALF. Eos, eosinophil; Lym, lymphocyte; Mac, macrophage; Neu, neutrophil, n = 6-8. (L) IL-5 in BALF (left) and lung (right) were determined by ELISA, $n=6(\mathrm{M})$ Lung eosinophil $\left(\mathrm{CD} 45^{+} \mathrm{CD}_{11 \mathrm{c}^{-} \text {SiglecF }}{ }^{+}\right)$numbers were assessed by flow cytometry as in Figure $1 \mathrm{C}, \mathrm{n}=6-8$. Level of (N, O) 116 mRNA and IL-6 protein in lung and (P) Gob5 mRNA in the lungs were assayed by qPCR and ELISA, respectively. $\mathrm{n}=6-8$. Data were pooled from 2 independent experiments. F4/80: adhesion G-protein-coupled receptor E1. SP-C: Surfactant protein C. ${ }^{*} P<.05$, ${ }^{* *} P<.01,{ }^{* * *} P<.001$ and ${ }^{* * * *} P<.0001$
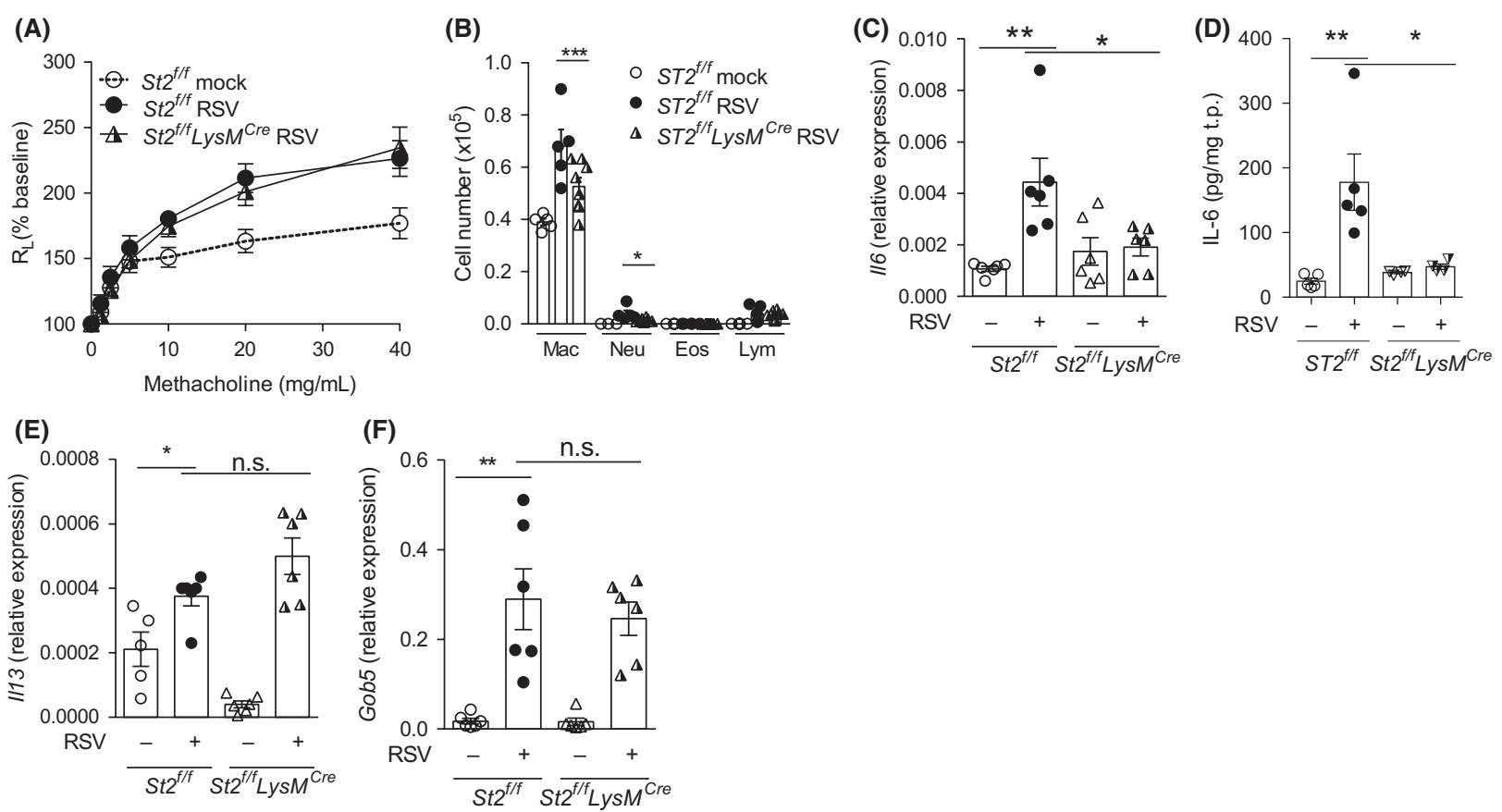

FIGURE 5 Myeloid cells facilitate RSV-induced airway inflammation in a ST2-dependent manner. St $2^{f / f}$ LysM ${ }^{\text {cre }}$ and St ${ }^{f / f}$ littermate mice were infected with RSV or mock and sacrificed 6 d post-infection. $(A)$ Changes in lung resistance $\left(R_{L}\right) n=4-6$. (B) Cellular composition in the BALF (Eos, Eosinophil; Lym, Lymphocyte; Mac, Macrophage; Neu, Neutrophil) of mice after infection. $n=5-8$. (C-F) mRNA expression of (C) II6, (D) IL-6 protein, (E) II13, and (F) Gob5 in the lungs of mice after infection. $n=5-6$. Data were pooled from 2 independent experiments. ${ }^{*} P<.05,{ }^{* *} P<.01$, and ${ }^{* * *} P<.001$

results suggest a positive correlation between RSV infection and lung cell death.

\section{5 | RSV-driven pulmonary IL-33 evokes circulating eosinophilia through ILC2s}

Peripheral blood eosinophil count during RSV bronchiolitis is a predictive factor of wheezing illness. ${ }^{38}$ Nevertheless, how RSV triggers circulating eosinophilia remains uncharacterized. To this end, we first analyzed the blood eosinophil profile of RSV-infected mice (Figure 6A,C). We found that the percentages of blood eosinophils peaked on day 9 post-RSV infection, but declined on day 14. Notably, blood eosinophilia was impaired in $1133^{f / f} \mathrm{LysM}^{\mathrm{Cre}}$, $1133^{-\%}$, and YetCre-13 Rosa-DTA mice on day 9 post-infection (Figure 6B,C). Taken together, these results suggest that RSV infection drives circulating eosinophilia in mice, and myeloid IL-33 and ILC2s contribute to this phenomenon.

\section{4 | DISCUSSION}

In this study, we demonstrated that IL-33 exerted diverse functions under RSV infection. RSV could trigger IL-33 production from 
(A) $\underline{\text { Blood }}$

\section{RSV}

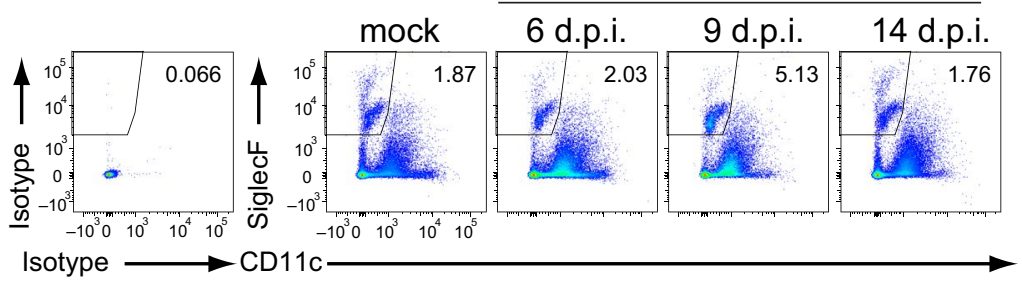

(B)
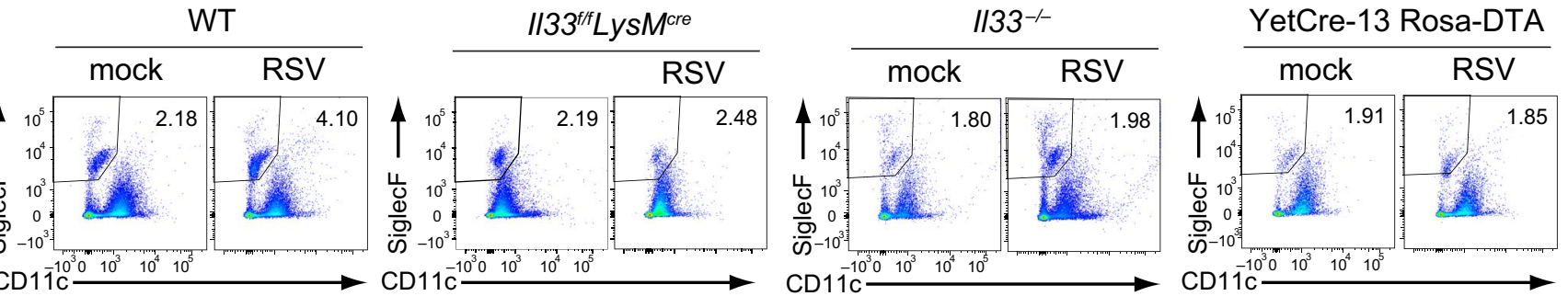

(C)

Blood eosinophils (CD11c SiglecF ${ }^{+}$)

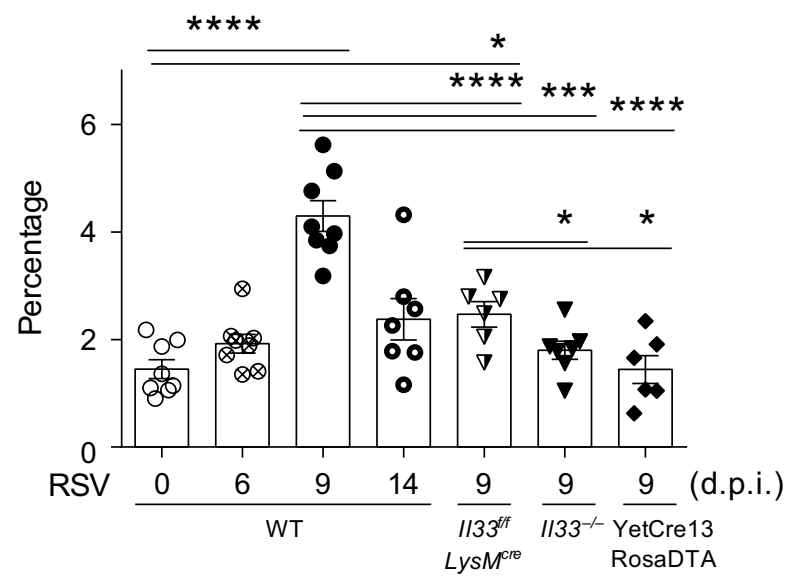

FIG URE 6 RSV-driven pulmonary IL-33 is required to evoke systemic eosinophilia. (A) Wild-type mice were infected with RSV (L19, $10^{6}$ pfu), and blood samples were harvested at the indicated time points. (B) $I 133^{f / f} \mathrm{Lys}^{\mathrm{cre}}, 1 / 33^{-/-}$, and YetCre-13 Rosa-DTA were infected with RSV, and blood samples were harvested on 9 d.p.i.. (A-B) Representative flow cytometry plot showing blood eosinophils (CD45 ${ }^{+}$CD11c $^{-}$ Siglec $\mathrm{F}^{+}$). (C) Percentage of blood eosinophils in previously described mice. Data were merged data from 2 independent experiments $\mathrm{n}=6-8$. ${ }^{*} P<.05,{ }^{* * *} P<.001,{ }^{* * * *} P<.0001$

multiple sources, including lung structural cells and myeloid cells. Global knockout of IL-33 resulted in reduced Th2 cytokine production from ILC2s, supporting the role of ILC2 in IL-13 production and AHR. We also showed that IL-33 produced by myeloid cells was required for IL-6 production in the lungs and airway neutrophilia in the respiratory tract during RSV infection. Lastly, both IL-33 derived from myeloid and structural cells contributed to eosinophilia in the lung and periphery. Collectively, our study suggests a differential, but complementary roles of IL-33 from different sources in driving airway inflammation during RSV infection.

IL-33 is a cytokine that can boost airway inflammation, mucus production, and Th2 cytokine production in the lungs under influenza virus, RSV, and rhinovirus infection. ${ }^{25,39-41}$ By disrupting IL-33 signaling through deletion, we found that IL-33 is indispensable for AHR and BALF cellularity driven by RSV infection. This is in agreement with a previous study showing that anti-ST2 antibody treatment suppressed RSV-induced $\| 5$ and $\| 13$ mRNAs in the lungs and BALF. ${ }^{40}$ Nevertheless, there are conflicting reports on the importance of IL-33 during RSV infection. For instance, Stier et al demonstrated that TSLP, but not IL-33, is the major cytokine that triggers type- 2 response through ILC2 under RSV infection. ${ }^{29}$ Additionally, Saravia et al showed that IL-33-ILC2 axis is activated in neonates but not adults. ${ }^{34}$ One possible explanation for this discrepancy is the different RSV strains used. Stier et al used 01/2-20 strain and found that only IL-13, but not IL-5, was induced in the lung. In our study, we used L19 strain, which was previously shown to induce IL-13. ${ }^{15}$ Here, we further demonstrate that IL-5 can be induced in the lungs by L19 strain. Furthermore, L19 strain induces robust Th2 inflammation characterized by AHR and mucus hypersecretion compared to other strains of the same antigenic subgroup like A2 and Long strains. ${ }^{15,42}$ Therefore, different RSV strains could exert different signaling mechanism that affects the overall inflammatory 
phenotype. Above all, we showed the cause-effect relationship between IL-33-ILC2 axis and L19-mediated type-2 inflammation.

Although previous study has implicated type-2 pneumocytes and other structural cells as the major producers of IL-33 in the lungs, ${ }^{43}$ other studies have detected IL-33 in myeloid cells under various allergen challenge and during viral infection. ${ }^{25,28,44}$ Moreover, a recent study in II33-driven citrine reporter mice have demonstrated that $\mathrm{CD} 45^{+}$cells expressed low levels of IL-33 at steady-state, and allergen exposure augmented its expression. ${ }^{45}$ In the present study, we observed that RSV-induced IL-33 expression in both myeloid and lung structural cells. We further investigated the pathological roles of myeloid cell-derived IL-33 and ST2 signaling by using myeloid cellspecific knockout mice. We observed RSV-induced lower neutrophilia in both myeloid-specific IL-33-deficient (II33 ${ }^{f / f}$ LySM $^{\mathrm{Cre}}$ mice) and ST2deficient ( $S t 2^{f / f} L_{y s} M^{C r e}$ mice) mice, compared to their WT littermates, suggesting that myeloid cell-derived IL-33/ST2 signaling is critical for RSV-induced airway neutrophilia. Nevertheless, it was worth noting that AHR, the expression levels of II13 and the associated Gob5 were unaffected in the St2 $2^{f / f}$ LysM $^{\text {Cre }}$ mice, suggesting that unlike ILC2s, ST2 ${ }^{+}$ myeloid cells are dispensable for RSV-induced AHR. Taken together, myeloid cell-derived IL-33 contributes to RSV-driven pathogenesis, and the $\mathrm{ST}^{+}$myeloid cells are responsible for airway neutrophilia.

In line with airway neutrophilia, RSV-induced IL-6 production was also suppressed when myeloid IL-33 or ST2 was depleted. Indeed, IL-33 has been shown to drive IL-6 production by various myeloid cells, such as macrophages, mast cells, and DC, contributing to tissue inflammation. ${ }^{46}$ Moreover, studies have shown that IL-6 signaling can induce neutrophil recruitment to the lungs under various stimulation including allergen ${ }^{47}$ and endotoxin. ${ }^{48}$ IL- 6 can boost neutrophil numbers through various ways, including suppression of apoptosis ${ }^{49}$ and sensitizing neutrophils toward chemokine cues like $\mathrm{IL}-8 .{ }^{50}$ Therefore, the observed reduction in airway neutrophilia in our study could be a direct consequence of reduced IL- 6 production due to impaired IL-33 production and ST2 signaling in myeloid cells. Nevertheless, the mechanism by which IL- 6 affects neutrophilia during RSV infection warrants further investigation.

Pulmonary eosinophilia and elevated lung IL-5 levels are features of RSV infection, with some studies suggesting that they are required for airway inflammation in murine models. ${ }^{23,51}$ Clinically, BALF IL-5 positively correlates to eosinophil level in PBMC. ${ }^{12}$ RSVdriven eosinophil activity positively correlates to wheezing illness in patients. ${ }^{38,52}$ In addition, increased systemic eosinophil activity has been reported in RSV-infected patients after discharge, ${ }^{53}$ although the immunoregulatory mechanism is unclear. In accordance with these findings, we observed eosinophil infiltration in the BALF, lungs, and periphery after RSV infection. Kinetically, eosinophilia was induced after the onset of AHR, in agreement with the aforementioned studies. Importantly, we also found that both myeloidderived IL-33 and ILC2 were necessary for RSV-driven IL-5 induction and peripheral eosinophilia. Depletion of IL-13-producing cells, predominantly ILC2, also reduced IL-5 and circulating eosinophilia. These results suggest that IL-33-ILC2 axis is crucial for RSV-driven type-2 inflammation.
In summary, we highlighted the importance of IL-13 produced by IL-33-activated ILC2s in triggering RSV-driven airway inflammation and the possible role of IL-5 in eliciting eosinophilia in the periphery under local IL-33 stimuli. These findings may explain how RSV triggers airway inflammation in the early phase of infection, before the initiation of adaptive immunity. Taken together, our results confirmed the pivotal role of myeloid IL-33 and ILC2 in RSV-driven IL-5 and eosinophilia. In addition, we found a novel mechanism by which myeloid cell-derived IL-33 and ST2 signaling contribute to IL-6 production, leading to the development of airway inflammation (Figure S6).

\section{ACKNOWLEDGMENT}

We would like to thank the staff of the IBMS Flow Cytometry Core Facility (AS-CFII-108-113) for services and facilities provided.

\section{CONFLICT OF INTEREST}

The authors have declared that no conflict of interest exists.

\section{AUTHOR CONTRIBUTIONS}

YHW planned and performed experiments and wrote the manuscript. ACYL performed experiments, prepared the materials, and wrote the manuscript. PYC performed experiments and prepared the materials. CLPT performed experiments and edited the manuscript. WYC provided the IL-33-knockout, IL-33-floxed mice, St2floxed mice and provided technical advises on IF staining. $L Y L$ and CHT provide clinically relevant information about RSV. NWL provided the RSVs and advised on their propagation. YJC conceived and initiated the project, planned experiments, and wrote the manuscript.

\section{ORCID}

Yi-Hsiu Wu iD https://orcid.org/0000-0002-4385-2532

Ching-Hui Tsai iD https://orcid.org/0000-0003-0998-8230

Yungling Leo Lee (iD https://orcid.org/0000-0002-2234-9479

Ya-Jen Chang (iD https://orcid.org/0000-0003-1377-6142

\section{REFERENCES}

1. Martinez FD. Respiratory syncytial virus bronchiolitis and the pathogenesis of childhood asthma. Pediatr Infect Dis J. 2003;22(2 Suppl):S76-S82.

2. Falsey AR, Hennessey PA, Formica MA, Cox C, Walsh EE. Respiratory syncytial virus infection in elderly and high-risk adults. N Engl J Med. 2005;352(17):1749-1759.

3. Sigurs N, Aljassim F, Kjellman B, et al. Asthma and allergy patterns over 18 years after severe RSV bronchiolitis in the first year of life. Thorax. 2010;65(12):1045-1052.

4. Peebles RS Jr, Hashimoto K, Graham BS. The complex relationship between respiratory syncytial virus and allergy in lung disease. Viral Immunol. 2003;16(1):25-34. 
5. Arnold R, Konig B, Galatti H, Werchau H, Konig W. Cytokine (IL-8, IL-6, TNF-alpha) and soluble TNF receptor-I release from human peripheral blood mononuclear cells after respiratory syncytial virus infection. Immunology. 1995;85(3):364-372.

6. Tsutsumi H, Matsuda K, Sone S, Takeuchi R, Chiba S. Respiratory syncytial virus-induced cytokine production by neonatal macrophages. Clin Exp Immunol. 1996;106(3):442-446.

7. McNamara PS, Flanagan BF, Selby AM, Hart CA, Smyth RL. Proand anti-inflammatory responses in respiratory syncytial virus bronchiolitis. Eur Respir J. 2004;23(1):106-112.

8. Moreno-Solis G, Torres-Borrego J, de la Torre-Aguilar MJ, Fernandez-Gutierrez F, Llorente-Cantarero FJ, Perez-Navero JL. Analysis of the local and systemic inflammatory response in hospitalized infants with respiratory syncitial virus bronchiolitis. Allergol Immunopathol (Madr). 2015;43(3):264-271.

9. Mukherjee S, Lindell DM, Berlin AA, et al. IL-17-induced pulmonary pathogenesis during respiratory viral infection and exacerbation of allergic disease. Am J Pathol. 2011;179(1):248-258.

10. Krishnamoorthy $\mathrm{N}$, Khare $\mathrm{A}$, Oriss $\mathrm{TB}$, et al. Early infection with respiratory syncytial virus impairs regulatory $T$ cell function and increases susceptibility to allergic asthma. Nat Med. 2012;18(10):1525-1530.

11. Schwarze J, Hamelmann E, Bradley KL, Takeda K, Gelfand EW. Respiratory syncytial virus infection results in airway hyperresponsiveness and enhanced airway sensitization to allergen. J Clin Invest. 1997;100(1):226-233.

12. Kim CK, Kim SW, Park CS, Kim BI, Kang H, Koh YY. Bronchoalveolar lavage cytokine profiles in acute asthma and acute bronchiolitis. $J$ Allergy Clin Immunol. 2003;112(1):64-71.

13. Bertrand P, Lay MK, Piedimonte G, et al. Elevated IL-3 and IL-12p40 levels in the lower airway of infants with RSV-induced bronchiolitis correlate with recurrent wheezing. Cytokine. 2015;76(2):417-423.

14. Tekkanat KK, Maassab HF, Cho DS, et al. IL-13-induced airway hyperreactivity during respiratory syncytial virus infection is STAT6 dependent. J Immunol. 2001;166(5):3542-3548.

15. Lukacs NW, Moore ML, Rudd BD, et al. Differential immune responses and pulmonary pathophysiology are induced by two different strains of respiratory syncytial virus. Am J Pathol. 2006;169(3):977-986.

16. Peebles RS Jr, Hashimoto K, Collins RD, et al. Immune interaction between respiratory syncytial virus infection and allergen sensitization critically depends on timing of challenges. J Infect Dis. 2001;184(11):1374-1379.

17. Taylor G, Stott EJ, Hughes M, Collins AP. Respiratory syncytial virus infection in mice. Infect Immun. 1984;43(2):649-655.

18. Hoyler T, Klose CS, Souabni A, et al. The transcription factor GATA-3 controls cell fate and maintenance of type 2 innate lymphoid cells. Immunity. 2012;37(4):634-648.

19. Oliphant CJ, Hwang YY, Walker JA, et al. MHCll-mediated dialog between group 2 innate lymphoid cells and CD4(+) T cells potentiates type 2 immunity and promotes parasitic helminth expulsion. Immunity. 2014;41(2):283-295.

20. Halim TY, Krauss RH, Sun AC, Takei F. Lung natural helper cells are a critical source of Th2 cell-type cytokines in protease allergen-induced airway inflammation. Immunity. 2012;36(3):451-463.

21. Kim BS, Siracusa MC, Saenz SA, et al. TSLP elicits IL-33-independent innate lymphoid cell responses to promote skin inflammation. Sci Transl Med. 2013;5(170):170ra16-170ra16.

22. Klose CS, Artis D. Innate lymphoid cells as regulators of immunity, inflammation and tissue homeostasis. Nat Immunol. 2016;17(7):765-774

23. Liu J, Wu J, Qi F, et al. Natural helper cells contribute to pulmonary eosinophilia by producing IL-13 via IL-33/ST2 pathway in a murine model of respiratory syncytial virus infection. Int Immunopharmacol. 2015;28(1):337-343.
24. Gorski SA, Hahn YS, Braciale TJ. Group 2 innate lymphoid cell production of IL-5 is regulated by NKT cells during influenza virus infection. PLoS Pathog. 2013;9(9):e1003615.

25. Chang YJ, Kim HY, Albacker LA, et al. Innate lymphoid cells mediate influenza-induced airway hyper-reactivity independently of adaptive immunity. Nat Immunol. 2011;12(7):631-638.

26. Cayrol C, Girard JP. IL-33: an alarmin cytokine with crucial roles in innate immunity, inflammation and allergy. Curr Opin Immunol. 2014;31:31-37.

27. Liew FY, Girard JP, Turnquist HR. Interleukin-33 in health and disease. Nat Rev Immunol. 2016;16(11):676-689.

28. Qi F, Wang D, Liu J, et al. Respiratory macrophages and dendritic cells mediate respiratory syncytial virus-induced IL-33 production in TLR3- or TLR7-dependent manner. Int Immunopharmacol. 2015;29(2):408-415.

29. Stier MT, Bloodworth MH, Toki S, et al. Respiratory syncytial virus infection activates IL-13-producing group 2 innate lymphoid cells through thymic stromal Iymphopoietin. J Allergy Clin Immunol. 2016;138(3): 814-824.e11.

30. Qi F, Bai S, Wang D, et al. Macrophages produce IL-33 by activating MAPK signaling pathway during RSV infection. Mol Immunol. 2017;87:284-292.

31. Akbari O, Stock P, Meyer E, et al. Essential role of NKT cells producing IL-4 and IL-13 in the development of allergen-induced airway hyperreactivity. Nat Med. 2003;9:582-588.

32. Hansen G, Berry G, DeKruyff RH, Umetsu DT. Allergen-specific Th1 cells fail to counterbalance Th2 cell-induced airway hyperreactivity but cause severe airway inflammation. J Clin Invest. 1999;103:175-183.

33. Rudd BD, Schaller MA, Smit JJ, etal. MyD88-mediated instructive signals in dendritic cells regulate pulmonary immune responses during respiratory virus infection. J Immunol. 2007;178(9):5820-5827.

34. Saravia J, You D, Shrestha B, et al. Respiratory syncytial virus disease is mediated by age-variable IL-33. PLoS Pathog. 2015;11(10):e1005217.

35. Price $A E$, Liang $H E$, Sullivan $B M$, et al. Systemically dispersed innate IL-13-expressing cells in type 2 immunity. Proc Natl Acad Sci USA. 2010;107(25):11489-11494.

36. Clausen BE, Burkhardt C, Reith W, Renkawitz R, Forster I. Conditional gene targeting in macrophages and granulocytes using LysMcre mice. Transgenic Res. 1999;8(4):265-277.

37. Rincon M, Irvin CG. Role of IL- 6 in asthma and other inflammatory pulmonary diseases. Int J Biol Sci. 2012;8(9):1281-1290.

38. Ehlenfield DR, Cameron K, Welliver RC. Eosinophilia at the time of respiratory syncytial virus bronchiolitis predicts childhood reactive airway disease. Pediatrics. 2000;105(1):79-83.

39. Le Goffic R, Arshad MI, Rauch M, et al. Infection with influenza virus induces IL-33 in murine lungs. Am J Respir Cell Mol Biol. 2011;45(6):1125-1132.

40. Zeng S, Wu J, Liu J, Qi F, Liu B. IL-33 receptor (ST2) signalling is important for regulation of Th2-mediated airway inflammation in a murine model of acute respiratory syncytial virus infection. Scand $J$ Immunol. 2015;81(6):494-501.

41. Jackson DJ, Makrinioti H, Rana BM, et al. IL-33-dependent type 2 inflammation during rhinovirus-induced asthma exacerbations in vivo. Am J Respir Crit Care Med. 2014;190(12):1373-1382.

42. Stokes KL, Chi MH, Sakamoto K, et al. Differential pathogenesis of respiratory syncytial virus clinical isolates in BALB/c mice. J Virol. 2011;85(12):5782-5793.

43. Yasuda K, Muto T, Kawagoe T, et al. Contribution of IL-33-activated type II innate lymphoid cells to pulmonary eosinophilia in intestinal nematode-infected mice. Proc Natl Acad Sci USA. 2012;109(9):3451-3456.

44. Su Z, Lin J, Lu F, et al. Potential autocrine regulation of interleukin-33/ST2 signaling of dendritic cells in allergic inflammation. Mucosal Immunol. 2013;6(5):921-930. 
45. Hardman CS, Panova V, McKenzie AN. IL-33 citrine reporter mice reveal the temporal and spatial expression of IL-33 during allergic lung inflammation. Eur J Immunol. 2013;43(2):488-498.

46. Afferni C, Buccione C, Andreone S, et al. The pleiotropic immunomodulatory functions of IL-33 and its implications in tumor immunity. Front Immunol. 2018;9:2601.

47. Chu DK, Al-Garawi A, Llop-Guevara A, et al. Therapeutic potential of anti-IL-6 therapies for granulocytic airway inflammation in asthma. Allergy Asthma Clin Immunol. 2015;11(1):14.

48. Miyamoto $M$, Prause $O$, Sjostrand M, Laan M, Lotvall J, Linden A. Endogenous IL-17 as a mediator of neutrophil recruitment caused by endotoxin exposure in mouse airways. J Immunol. 2003;170(9):4665-4672.

49. Asensi V, Valle E, Meana $A$, et al. In vivo interleukin-6 protects neutrophils from apoptosis in osteomyelitis. Infect Immun. 2004;72(7):3823-3828.

50. Wright HL, Cross AL, Edwards SW, Moots RJ. Effects of IL-6 and IL-6 blockade on neutrophil function in vitro and in vivo. Rheumatology (Oxford). 2014;53(7):1321-1331.

51. Schwarze J, Cieslewicz G, Hamelmann E, et al. IL-5 and eosinophils are essential for the development of airway hyperresponsiveness following acute respiratory syncytial virus infection. J Immunol. 1999;162(5):2997-3004.
52. Zhao J, Takamura M, Yamaoka A, Odajima Y, likura Y. Altered eosinophil levels as a result of viral infection in asthma exacerbation in childhood. Pediatr Allergy Immunol. 2002;13(1):47-50.

53. Lindemans CA, Kimpen JL, Luijk B, et al. Systemic eosinophil response induced by respiratory syncytial virus. Clin Exp Immunol. 2006;144(3):409-417.

\section{SUPPORTING INFORMATION}

Additional supporting information may be found online in the Supporting Information section.

How to cite this article: Wu Y-H, Lai AC-Y, Chi P-Y, et al; Pulmonary IL-33 orchestrates innate immune cells to mediate respiratory syncytial virus-evoked airway hyperreactivity and eosinophilia. Allergy. 2020;75:818-830. https://doi. org/10.1111/all.14091 